\title{
Essais
}

ESSAIS

Revue interdisciplinaire d'Humanités

$7 \mid 2015$

Normes communiquées, normes communicantes

\section{Modifications corporelles dans l'art contemporain occidental actuel : une certaine transgression}

Catherine Souladié

\section{OpenEdition}

1 Journals

Édition électronique

URL : http://journals.openedition.org/essais/6330

DOI : $10.4000 /$ essais. 6330

ISSN : 2276-0970

Éditeur

École doctorale Montaigne Humanités

Édition imprimée

Date de publication : 1 décembre 2015

Pagination : 86-95

ISBN : 978-2-9544269-6-9

ISSN : 2417-4211

Référence électronique

Catherine Souladié, « Modifications corporelles dans l'art contemporain occidental actuel : une certaine transgression », Essais [En ligne], 7 | 2015, mis en ligne le 26 février 2021, consulté le 27

février 2021. URL : http://journals.openedition.org/essais/6330 ; DOI : https://doi.org/10.4000/essais. 6330 


\title{
Modifications corporelles dans l'art contemporain occidental actuel : une certaine transgression
}

\author{
Catherine Souladié
}

De tous les matériaux, lointains et intimes, abstraits ou vécus, le corps humain entraine hors de méthodologies établies et des théories toutes faites. Pour paraphraser Roland Barthes lorsqu'il parle du "moi ", le corps embrasse tous les lieux du possible qu'aucune contradiction n'effraie et devient ainsi la plus haute ambition de la vie. Dans les arts plastiques actuels, il est des pratiques où le corps humain se pose en révélateur et catalyseur, permettant d'atteindre les limites des possibles, et ainsi éclaire des zones d'ombre au cœur de la création et de ses liens avec le social. Le corps est la préoccupation de notre temps : de nombreux langages corporels transgressent, et paradoxalement, effacent sans arrêt ce corps dans nos sociétés occidentales. Il est ici question de sa présence.

Dans l'Histoire de l'art, le corps a souvent hérité d'un rôle secondaire, son expressivité étant confinée aux territoires des « bio-pouvoirs » définis par Michel Foucault, techniques spécifiques du pouvoir s'exerçant sur les corps individuels et les populations, comme si la structure du corps collectif était plus importante que toute destinée individuelle, avec une possible et dangereuse transformation de l'individu. Au XXe siècle, le corps émerge comme le point central d'une immensité de signes porteurs de modifications, d'ouvertures et d'inattendus. Les corps inscrits, œuvres et sujets des arts plastiques, placent d'emblée certains artistes dits d'attitude ainsi que le spectateur dans une position divergente : il s'agit d'un "corps à corps " avec l'art, au sein duquel l'individu seul devient cible de changement et de métamorphose. L'art d'attitude se constitue dans une espèce d'écart, de désobéissance, d'insoumission. La dimension scénique du travail suppose aussitôt un recours à l'excès. Dans cette vision, tous les aspects vitaux du corps sont envisagés : corps intime, corps souffrant, corps salis, corps mourant, corps jouant, corps laid, corps sexuel. Les happenings des années soixante doivent beaucoup aux Dadaïstes parisiens des années vingt : l'Art Corporel ou "sculpture vivante " reste encore aujourd'hui redevable de Duchamp se tonsurant. C'est donc le 
corps de l'artiste qui fait l'œuvre. Exclusif, arrogant, intransigeant, cet art naît de la prise en compte du corps comme "donnée fondamentale » se générant du désir d'un corps retrouvé, avènement d'une nouvelle relation sociale, désaliénée, fortement libertaire. Le Manifeste pour l'Art Corporel, rédigé par le critique François Pluchard ${ }^{1}$ et daté de décembre 1974 l'annonçait déjà. En 1973, l'artiste italienne Gina Pane inscrit son corps d'une Action sentimentale, s'entaillant la peau avec une lame de rasoir pour mieux s'ouvrir au monde. Automutilée au rasoir pour imprimer dans sa chair une étoile de sang (The Star, 1975), Marina Abramovic inscrit ainsi la passion et la mise en danger à-même le corps. Des gestes, aux références politiques et chamaniques, qui relèvent d'une expressivité primale : le corps, tel un basique tracé à la sanguine, ici dernière frontière de la dépossession individuelle, comme territoire d'une inscription ultime renvoyant paradoxalement aux origines de l'écriture, et donc de l'art. Par un puissant effet de levier dramatique, la stricte économie de ces signes radicaux éprouve de manière saisissante les limites du langage, mais aussi la responsabilité de notre regard passif sur la violence. Mais ici encore, le corps reste le lieu d'inscription, le support provocateur contre une esthétique qui voudrait que l'art ne soit que beau... Si le corps s'ouvre au sens propre dans les arts plastiques, les peaux, toiles vierges, se taguent et se graphent.

Si nous suivons quelques définitions basiques : le tatouage " est l'action d'ouvrir la peau et d'y déposer des pigments de manière à fixer un dessin dans la cicatrice $»^{2}$; le piercing est "l'action de percer la peau de manière à créer un canal pouvant accueillir un bijou $"^{3}$ ou autre implant... Une réminiscence de ces rituels dits archaïques, à l'image de Fakir Musafar et ses Primitifs Modernes des années 1980, annonce donc un retour dans la société occidentale de modifications corporelles d'autres époques et d'autres cultures : la « contorsion " (étirement du corps), la " constriction " (compression du corps), la "déprivation» (restriction de mouvement, isolement dans des cases), l' " encombrement " (suspension de poids sur diverses parties du corps visant à l'allongement), la " pénétration " (piercings, planches à clous), la " brûlure " (marquage au fer rouge), la «suspension » (à l'aide de crochets) seront désormais le vocabulaire des artistes "perceurs-tatoueurs-scarificateurs ». Nos sociétés actuelles se mettent à l'écoute des primitivismes, et ces marquages, désormais démocratisés, annoncent un corps labile rhizomant vers des variations de l'apparence. Un tel renouvellement ne ramène pas au tribalisme mais ouvre vers un éclectisme cosmétologique postmoderne.

\footnotetext{
1 Voir son ouvrage de référence : François Pluchard, L'Art corporel, Paris, Limage 2, 1983.

2 Source : http://www.ens-louis-lumiere.fr/fileadmin/recherche/Caffier-photo-2008-mem-annexes. pdf [consulté le 04/05/2014].

Ibid.
} 


\section{Albrecht Becker, « le déviant-fondateur »}

En effet, déviant ces rituels, quelques pratiques plastiques actuelles affirment reprendre le contrôle du corps humain en le modifiant et cultivant ainsi la peau : l'addition d'une certaine artificialité amène un nouvel ordre au-delà de toute valeur esthétique. Parlons du précurseur Albrecht Becker, artiste allemand (1906-2002), persécuté par les Nazis, et avec lequel prend forme une grammaire corporelle atypique au style kitsch. Il semble en fait que sa souffrance du départ, relative à son homosexualité établie dans un univers social qui ne l'admet pas, révèle un cheminement entre la persécution causée par la guerre et son travail sur le corps. Dès les années 1940, il envisage le tatouage comme corps/décor, ne cessant de se tatouer et "sur-tatouer ", jusqu'à se faire lui-même des traits à la lame de rasoir et badigeonner de l'encre dans les quelques morceaux restés vierges.

"Très vite son corps devint la partition d'un crescendo qu'il ne souhaite (ne peut) plus arrêter. Des chevilles jusqu'aux poignets et à l'encolure du cou, il n'est pas un bout d'épiderme qui ne soit tatoué. Les plis qui marquent le vieillissement font évoluer les formes et les couleurs des tatouages, semblant leur faire prendre une trajectoire autonome qui dépasse les seules intentions de Becker » . Prune Chanay, auteur d'un article «Becker le marqué », poursuit: «Son corps comme un palimpseste, est devenu libido, un livre ouvert où les écritures se libèrent et se superposent, brouillant les pistes en empêchant toutes lectures univoques : son corps illustre de manière toujours différente une seule et même histoire, la sienne, abolissant les notions de dehors et de dedans, le dehors étant un autre dedans $"$ ". Le corps devient alors une œuvre d'art authentique, évoluant seule, sans son auteur, tel un objet-tableau, ou même un ouvrage littéraire dont on découvrirait la langue. Bien au-delà d'une jouissance de la douleur relative à la lame tranchant son épiderme, Becker, victime et bourreau de lui-même, et loin des pratiques sadomasochistes habituelles, prend possession de son corps par le tatouage, tel un prolongement sur la matière-peau : les tatouages de son pénis, tel un ersatz de sexualité, ou les injections de litres de paraffine dans ce même membre afin de l'aplanir en triangle pubien (ce qui a donné une masse définitive au niveau de son sexe) font de son corps un territoire d'évasion, domaine ambivalent de souffrance/jouissance, et orgasme/vie. De plus, on reconnait un sexe percé dans ce moignon tuméfié : Albrecht Becker écrit le livre vierge de sa sexualité et confectionne ses " totems ", substituts, en cuir, du sexe "abîmé ". Il transcende ainsi une sexualité normative, le membre entouré d'une couronne d'épines ${ }^{6}$. Selon la théorie foucaldienne d'une imbrication

4 Prune Chanay, «Becher le marqué », Quasimodo, «Modifications corporelles », n 7, Montpellier, Association Quasimodo et Fils, 2003, p. 105-112.

5 Ibid., p. 111.

6 Les images concernant les pratiques d'Albrecht Becker sont visibles sur le site : http://hervejosephlebrun.wordpress.com/articles-de-presse/ [consulté le 04/05/2014]. 
certaine entre pouvoir et sexualité, Becker montre ici une autonomie de jouissance passant par le tatouage et la perforation de son corps, comme un prolongement sur la matière ; il écrit et s'inscrit dans une "contre-sexualité " selon l'expression de la philosophe Beatriz Preciado ${ }^{7}$, pratique perçue comme déviante et improductive, cumulant une tentative de l'entre-deux genres et une remise en cause de l'intégrité physique du corps humain. Après Duchamp, et à l'instar de son contemporain Pierre Molinier, Becker se fait sujet et objet de son œuvre; mais à la différence de ses deux conferes, il prend possession de son corps en jouant du couteau. Telle une Orlan à l'Euvre-Chair, il découvre un corps à la chair "dé-mesurée ". Ainsi la peau écrite d'Albrecht Becker passerait-elle le temps, recherchant une authentique liberté par une esthétique nouvelle et dérangeante. L'artiste semble nous dévoiler avec fierté ce nouveau corps bijou-polychromé. Avec une telle liberté de mouvement et subversion des formes, il fait de sa chair le lieu de création d'un métalangage, usant et abusant du procédé culturel récurrent qu'est le tatouage, et réinvente ainsi sa condition humaine. Dès l'après-guerre, à une époque quasiment vierge du corps vivant exposé, et tel un éclaireur, il interroge la difficulté de vivre, la notion de révolte et les limites de la création. Cependant, il n'y a pas ici de prosélytisme dans cette marginalité supposée du corps présentant une interface qui enrichit les notions de frontière et de contenant et qui déflore les prémisses de la «théorie Queer »8.

\section{Ron Athey, « le trans- gresseur »}

Le performer californien Ron Athey, plasticien des chairs, homosexualité et séropositivité revendiquées, se distingue par une altération relative et publique de la peau, l'assouplissant à coup de scarifications et d'incisions, et l'intégrant ainsi dans un univers symbolique traduisant un imaginaire de mutation. Athey graphe sa peau au gré de ses humeurs et désirs : elle est son journal intime, sa garde-robe et son garde-fou durant ses performances. Il parle de coquille et d'armure, entre un exhibitionnisme outrancier et un corps messager. Militant pour toutes causes perdues, chaman de la génération piercing, pratiquant le "cutting ", à savoir la lacération et le tatouage sur scène de son corps et de celui d'autrui, il multiplie les performances montrant couronnes d'aiguilles, sutures de lèvres et autres perçages de scrotums, proposant ainsi une vision

7 Beatriz Preciado, Manifeste contra-sexuel, traduit de l'espagnol par Marie-Hélène Bourcier, Paris, Éditions Balland, 2000.

8 Il s'agit, dans le travail d'Albrecht Becker, d'un travestissement initiatique de la théorie Queer, définie par les philosophes américaines contemporaines Judith Butler ou Monique Wittig (années 1990), suite aux travaux de Michel Foucault sur l'Histoire de la Sexualité : la question de l'identité est repensée ainsi que les liens entre sexe et genre ; le genre est une production performative, sans fondement ultime et essentiel, car produit par une incessante " chaîne citationnelle "; s'il est construit, le genre peut être donc déconstruit, via le travestissement par exemple. Voir Judith Butler, Gender Trouble, New York, Routledge, 1990. 
érotico-pornographique, radicale et extrême de l'Art Corporel. Transpercé tel un Saint-Sébastien du XXI ${ }^{\mathrm{e}}$ siècle, il hérite d'un art politique, sociologique, à la manière de l'Actionnisme Viennois et de Michel Journiac, revendiquant le corps comme un ultime recours. Ron Athey crée ses propres contes et légendes, baignés de fantasmes adolescents (Marlène), et de dieux récupérés dans divers cultes et religions. Une de ses créations et créatures fantastiques s'intitule "Hatchet Pussy " et relève d'un démon orné d'un soleil noir, étrangement semblable à l'idole satanique contenue dans les images de l'Apocalypse de SaintSever ${ }^{10}$. Athey a lu Georges Bataille et sa nouvelle intitulée l'Anus Solaire. Bataille écrit :

À ce moment, les hommes chercheront la mort et la mort les fuira. [...] On verra alors des sortes de locustes semblables à des chevaux équipés; elles auront des sortes de couronnes d'or sur la tête, et leur visage sera semblable au visage humain. [...] Elles auront des cuirasses semblables aux cuirasses de fer [...]. Elles auront des queues comme les scorpions et ces queues seront armées de dards ${ }^{11}$.

Si l'on écoute les croyances adventistes du XIX ${ }^{e}$ siècle rattachées à l'interprétation de l'Apocalypse de Saint Sever (écrite au VIII siècle), un deuxième messie s'annonce : Athey s'y prépare, et à son habitude, se sacrifie. Pourquoi pas lui ? Il peaufine donc sa mythologie personnelle. À l'instar de la déesse "Kâlî, la Noire ", mère destructrice et créatrice de l'hindouisme, image ultime de l'annihilation, mais aussi, ultime réalité et source de l'être dans le cadre des croyances tantriques, la déesse d'Athey est une princesse prostituée au visage agrafé (Staple), au sexe cousu (Hatchet Pussy) et à quatre bras : deux mains et deux godemichets ou dildos. Le nouveau messie serait donc Queer, autocentré, et inscrit un coït parodique dans un monde purement carnavalesque, lequel semble indiquer une refonte du corps, de ses organes et de ses modèles.

En effet, par ce mélange des genres entre tribalisme reconstitué, archaïsme réinventé, et une esthétique de jeu de jambes via la créature mi Queer-mi poupée de Pierre Molinier, Athey rentre ici dans une fiction hétérosexuelle "Queer» : cela recouvre alors des pratiques de re-significations et de recodifications antihégémoniques et performatives, dont le but est de redéfinir des espaces de

9 Anus solaire, Staple-Hatchet Pussy, photographies par Catherine Opie visible sur le site : http: //www.ecrans.fr/Le-corps-contamine-de-Ron-Athey,2311.html ; l'œuvre de Ron Athey est visible sur les sites : www.ronathey.com et http://ronatheynews.blogspot.fr/ [consultés le 04/05/2014].

10 Apocalypse de Saint-Sever: Satan et les anges déchus font srabattre sur la Terre des sauterelles, dont certaines ont un corps de cheval. Miniature extraite de l'Apocalypse de Saint-Sever (1028). Source : http://www.larousse.fr/encyclopedie/image/Apocalypse_de_Saint-Sever/1009332 [consulté le 04/05/2014].

11 Georges Bataille, "L'Apocalypse de Saint-Sever ", in Euvres complètes, premiers écrits 19221940, Paris, Gallimard, 1970, p. 169. 
résistance aux régimes de la normalité ${ }^{12}$. La recodification de sa peau, par le tatouage et le piercing, permet la mise en place d'une technologie prothétique, remettant en cause les modèles cartésiens corps/esprit, nature/technologie, valide/handicapé, sain/malade, ou femme/homme. La question est ici le choix d'un sexe autant que celui d'un corps. Il met alors en place un procédé qui promettrait la reconstruction d'un corps : son corps masculin (organe sexuel inclus) pouvant être construit prothétiquement, re-construit, déplacé voire remplacé. Athey démontre alors que le système biologique et les préceptes de la communication fonctionnent sur des logiques différentes. L'artiste trahit par sa pratique une perversion nécessaire pour une nouvelle donne éthique et morale, et illustre ainsi Saint-Foucault ${ }^{13}$ et son choix de ne plus penser le déterminisme sexuel dans sa seule perspective biologique. Cependant, la finalité de telle performance n'est pas dans une radicalité de défaire le genre, ou de dénaturaliser les lois des genres inscrites dans le corps, mais plus de déranger les habitudes culturelles du corps genré, selon l'approche développée par Judith Butler. L'envie irait en effet plutôt vers la génération de genres dissidents, en inventant de nouveaux styles de corps et d'autres modes signifiants, à savoir produire de nouveaux corps chimériques à travers des créations « contra-sexuelles ». Si l'œuvre d'Athey participe de la refondation de la sexualité, sa pratique relève d'une définition pornographique, dans le sens où elle témoigne d'un insolent et radical usage de la liberté. Athey s'inscrit dans une " histoire de l'art [...], celle de l'infinie liberté humaine, telle qu'elle s'exprime et se réalise dans l'évolution infinie des formes symboliques. Faites de ruptures, [...] l'histoire de l'art est en réalité celle des scandales relevés [...] par la ruse de l'esprit. [...] Les artistes nous auraient fait progresser dans une divination esthétisante des parts maudites de l'être humain $»^{14}$. Bernard Lafargue parle à ce propos d'art " érotico-pornographique ", la tradition judéo-chrétienne ayant auparavant cherché à étouffer toutes velléités sexuelles dans les œuvres d'art.

Mais au-delà d'un corps sexualisé, militant et recodé, Ron Athey montre la diversité, la vaste distribution géographique (par les tatouages et rituels de toutes origines réappropriés) et la puissance de ce qu'est devenu cet Art Corporel que l'on peut appeler permanent. Sa renaissance à la fin du XX $\mathrm{X}^{\mathrm{e}}$ siècle prend de multiples significations. Les découvrir même à travers des pratiques difficiles pourrait être un moyen d'en apprendre davantage sur une société qui a

12 Un parallèle peut être fait avec le travail de l'acteur-performeur anglais Lazlo Pearlman, utilisant le corps nu et tel un outil de performance puissant, comme une force perturbatrice, et contestant, entre autres, les notions normatives de genre et des identités sexuelles. Site de Lazlo Pearlman : http://www.lazlopearlman.com/ [consulté le 04/05/2014].

13 David Halperin, Saint-Foucault, Paris, Epel, 2000.

14 Bernard Lafargue, "Érotisme de la pornographie / Pornographie de l'érotisme " (Avantpropos), Figures de l'art, "Nude or Naked ? Érotiques ou pornographies de l'art ", n 4, SaintPierre du Mont, Eurédit, 1999, p. 9-10. 
abandonné ses structures traditionnelles. Les adeptes des pièces d'Athey essaient aussi de se trouver une nouvelle identité tout en tentant de se reconnecter avec un soi primitif, disparu, perdu, et voulant découvrir leurs lointaines parentés.

\section{Lukas Zpira, ou le corps post-normé}

Malgré les tabous et morales, le corps devient transmutations et métamorphoses. L'heure n'est plus à l'esprit kamikaze punk mais à la médicalisation. Progressivement, les artistes du corps des années 2000 se " chirurgicalisent " pour mieux briser la norme plastique. En choisissant des modifications qui font de son visage un ajustement de références artistiques et esthétiques, l'artiste française Orlan a été la première à inverser la logique de ce qu'elle nomme « la chirurgie du bonheur ». Depuis, la technique des implants sous-cutanés aux États-Unis, telle celle pratiquée par la star des transplanteurs nord-américains Steve Haworth ${ }^{15}$, a explosé délibérément le cadre artistique. De même, comment le corps majestueux mais besogneux de Ron Athey se confrontet-il aujourd'hui au positiviste de l'implant Lukas Zpira ${ }^{16}$, néo-idole freak et "bod-moder " français, plasticien-performer actuel, chercheur, documentariste et photographe d'une subculture ? Les arts plastiques nous promènent à travers les évolutions physiques, psychologiques, et nous amènent à nous interroger sur la post-humanité et sur les motivations de l'homme, ou le surhomme de demain.

Son corps, véritable laboratoire plastique proposant performances et expositions, use de tatouages, piercings, scarifications, implants de silicone et pièces en titane afin de replacer et remplacer la peau. De telles lacérations proposent une véritable sémiologie des modifications corporelles.

Par opposition aux Primitifs Modernes qui travaillent sur des bases d'anthropologie tribale, les Body-hacktivistes (fusion de hackers et activistes), comme se définit Zpira, pratiquent, théorisent et/ou inventent des modifications corporelles avant-gardistes et prospectives, influencées par la culture manga, la bande dessinée, les films et la littérature de science-fiction : "les termes Body Hacktiviste et Body Hacktivisme sous-entendent la nécessité d'action et de prise en main de nos destinées par la volonté perpétuelle de se réinventer ${ }^{17}$. Utilisant pleinement les supports numériques pour transmettre son projet, l'activiste anatomique Zpira propose donc un "corps hacké " néo-naturel, baptisé " musée anthropologique cybernétique ", et recommence ainsi l'écriture d'un corps originel. Au-delà de la mode, il s'agit d'une construction de soi : on distinguera les pratiques de destruction dues à une souffrance intérieure

15 Images-œuvres de Steve Haworth : http://www.stevehaworth.com/wordpress/index.php/ steve-haworth-portfolio/3d-body-implants [consulté le 04/05/2014].

16 Images-œuvres de Lukas Zpira sur les sites : http://www.body-art.net/ et http://www.laspirale. org/ [consultés le 04/05/2014].

17 Lukas Zpira, Body Hacktivisme: http://www.body-art.net/, page d'accueil, op. cit. 
importante de celles qui s'inscrivent dans une prise en charge de soi-même. L'acte est ici réfléchi : on renouvelle la peau, laquelle mue, et se ravive telle une prothèse : une réelle ouverture du corps sans la revendication d'une quelconque souffrance. Nous assistons, selon le sociologue Philippe Liotard ${ }^{18}$, à une nouvelle prise en charge de soi, par un acte corporel inédit et inaugural : à nouveau, cela interroge à propos de l'automutilation, de la douleur, de l'esthétique, de l'intégrité physique et ouvre une réflexion sur les normes corporelles actuelles et la transgression sociétale. Michel Foucault l'écrivait déjà : « les monstres sont comme le bruit de fond, le murmure ininterrompu de la nature $»^{19}$. Zpira définit un corps qui n'est plus le siège d'une identité personnelle mais, pour reprendre le mot de Denis Baron, un " alter-ego modifié $\aleph^{20}$; une nouvelle humanité s'approche de nos corps, irrépressiblement en mutation. Il n'y a pas ici de brouillon à parachever, mais à construire un projet propre et inédit. Corps mixés de tribalisme et de technologie, Zpira et Haworth manient ceux-ci comme une interface, connexion entre les rituels anciens et futurs, l'environnement d'hier et d'aujourd'hui, social, politique et artistique. Cette artialisation du corps mutant néo-archaïque ouvre sans doute une nouvelle socialisation de la peau, avec des créations incessantes de codes artistiques incluant les substances corporelles humaines, artificielles et virtuelles. Alors un retour de l'archaïsme dans les pratiques plastiques corporelles mènerait à une mutation possible de l'homme en cyborg, vers un «nomadisme Korporel». Si l'on considère comme le signale David Le Breton dans son ouvrage Signes d'identité ${ }^{21}$ que ce marquage corporel est une façon d'emporter le corps au-delà de la mort, de stopper esthétiquement sa dégénérescence face à la vieillesse, les corps tatoués de Becker ou de Athey peuvent s'apparenter à une utopie de soi-même par leur monstruosité, leur côté grotesque, voire leur insupportabilité, à l'instar du corps virtuel, défiant de la sorte la notion traditionnelle de handicap. Si Zpira relie les deux cultures dans leur recherche éthique d'une liberté morphologique, c'est qu'il existe dans ces pratiques des doléances politiques et libertaires. Ces corps hackés et ultimes sont éminemment politiques dans le renversement de la corporéité traditionnelle, à l'exemple des corps-actionnistes dans les années 1960 et 1970 .

18 Philippe Liotard, "Lukas Zpira : le corps piraté ", in Lukas Zpira, Onanisme manu militari II, Saint-Raphaël, Hors éditions, 2005.

19 Michel Foucault, Les mots et les choses, Paris, Gallimard, 1966, p. 169, cité par Denis Baron, Corps et Artifices, de Cronenberg à Zpira, Paris, L'Harmattan, 2007, p. 188.

20 Denis Baron, op. cit., p. 188.

21 David Le Breton, Signes d'identité. Tatouages, piercings et autres marques corporelles, Paris, Éditions Métailié, 2002. 


\section{Conclusion}

Ce marquage du corps n'a pas d'autre possibilité que la sincérité ; sans elle, il se dégrade. De même que le crayonnage du corps de Becker n'est ni de l'exhibitionnisme ni de la complaisance. Ces pratiques difficiles sont certes déviantes mais pas immorales : l'immoral serait la dépravation, la dégradation de ce qui est moral, et même " amoral ». L'amoral, ou sans morale pré-définie, ou agissant indifféremment à celle-ci, est inattaquable et innocent, zone dans laquelle se situe Becker, en libertaire assumé ; l'immoral, agissant contrairement à des morales ou usant de son utilisation capricieuse, est abject, dépravé, débauché. Par sa radicalité, ces artistes se libèrent et nous libèrent du préjugé de l'humiliation comme de celui de la douleur. Cette construction symbolique doit être acceptée comme une réalité double et non comme un produit avec une intention symbolique délibérée. Certes, de telles pratiques artistiques proposent des corps, mais elles n'imposent aucun prototype et encore moins un modèle basé sur un principe de sélection : les artistes sont habités par une force centripète, dictée par une envie de dépasser certains tabous, plus que par une recherche post-humaine qui fera école. En somme, le corps biologique demeure, mais on peut dire qu'un deuxième corps s'écrit en s'inscrivant, engagé essentiellement dans la communication, dans la relation à l'autre, en s'affranchissant de la dictature des besoins et des instincts. De là naît cette indépendance, ce désir de construire sa propre enveloppe corporelle, creuset de la liberté vis-à-vis de l'ordre de la nature. Avec des singularisations incroyables, en fonction de notre histoire personnelle, tous nos vécus seraient donc enracinés dans ce deuxième corps, et non dans le corps organique. Athey ou Zpira, véritables geishas tatoués et percés, exacerbent ces travestissements sociaux ritualisés, ces corps tribaux dans leurs pratiques violentes pour mieux nous purger du destin tragique de la vie. Le philosophe Richard Shusterman parlait déjà en 1992 d'un Art à l'état vif2, à savoir une pensée du corps qui ne saurait se résumer par un simple projet conceptuel et critique, mais se situerait du côté de pratiques "somatiques " à visée " thérapeutique et méliorative " (selon les mots de Richard Shusterman) et de procédés artistiques corporels empiriques. Il définit depuis une philosophie somatique à propos du " corps vivant ", lequel est " loin d'être simplement le site où l'on peut afficher son éthos et ses valeurs sous une forme attrayante, le corps est aussi le médium permettant de mieux sentir et mieux agir $»^{23}$. "Penser en

22 Richard Shusterman, L'Art à l'état vif. La pensée pragmatiste et l'esthétique populaire, Paris, Éditions de Minuit, 1992.

23 Richard Shusterman, Conscience du corps. Pour une soma-esthétique, traduit de l'anglais par Nicolas Vieillescazes, Paris, Éditions de L'Éclat, 2007. Préface sur le site internet L'Éclat [en ligne] : http://www.lyber-eclat.net/lyber/shusterman/CC-introduction.html [consulté le 02/10/2014]. 
corps " pour reprendre l'expression du philosophe, permet de dépasser un corps trop idéalisé, donc par essence obsolète. Styliser son corps et l'inscrire de façon ultime tels ces artistes bod-moders sismographes aident à mesurer les bouleversements d'une époque et d'une société donnée. En effet, si l'on regarde le corps comme une mécanique de notre inconscient, celui-ci reste un médium fondamental, l'organe originel, telle une interface première de la pensée de notre environnement.

\author{
Catherine Souladié \\ EA 4426 MICA \\ Université Bordeaux Montaigne \\ cathysouladie@cegetel.net
}

\begin{abstract}
Résumé
Il s'agit ici de présenter une exaltation de la tragédie à travers des modes artistiques exprimant la passion et l'ivresse d'un corps ultime. Certains artistes corporels sismographes inscrivent un corps nouvellement lisible et signifiant par la pratique performative de tatouages, piercings, implants, maquillages ou travestissements : par et au nom du corps, l'artiste allemand Albrecht Becker annonce, le Performer américain Ron Athey dévie, ou le hacker du corps français Lukas Zpira manifeste. Le corps devient un lieu de débats où chacun pourrait reprendre la place dans la nature qu'il aurait perdue, et ce sous la forme qu'il souhaite.
\end{abstract}

\title{
Mots-clés
}

Art corporel, art hors-limites, tatouage, piercing, modifications corporelles.

\begin{abstract}
It is a question here of presenting an ecstasy of the tragedy through artistic modes expressing the passion and the drunkenness of an ultimate body. Certain seismographic physical artists register a recently readable and significant body by the performative practice of tattoos, piercings, implants, make-ups or disguises: by and in the name of the body, the German artist Albrecht Becker announces, the American Performance artist Ron Athey diverts, or the hacker of the French body Lukas Zpira shows. The body becomes a place of debates where each could take back the place in the nature which it would have lost, and it under the shape which he wishes.
\end{abstract}

\section{Keywords}

Body art, body hacking, tattoo, piercing, extreme performance art, body modifications. 